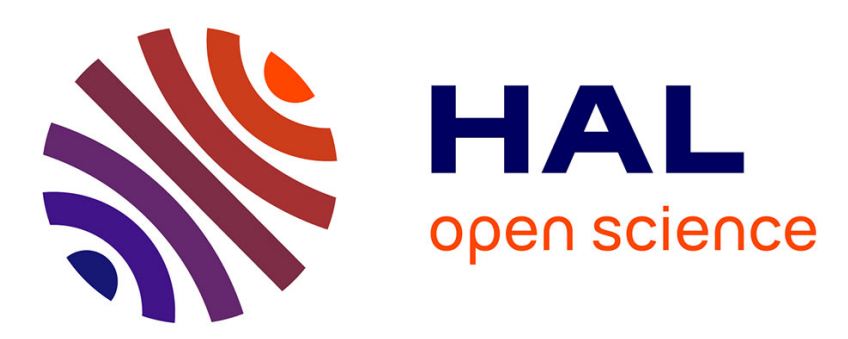

\title{
A novel predictive link quality metric for mobile ad-hoc networks in urban contexts
}

\author{
Sebastien Bindel, Serge Chaumette, Benoit Hilt
}

\section{To cite this version:}

Sebastien Bindel, Serge Chaumette, Benoit Hilt. A novel predictive link quality metric for mobile ad-hoc networks in urban contexts. 7th EAI International Conference on Ad Hoc Networks, Sep 2015, San Remo, Italy. 10.1007/978-3-319-25067-0_11 . hal-01277081

\section{HAL Id: hal-01277081 \\ https://hal.science/hal-01277081}

Submitted on 22 Feb 2016

HAL is a multi-disciplinary open access archive for the deposit and dissemination of scientific research documents, whether they are published or not. The documents may come from teaching and research institutions in France or abroad, or from public or private research centers.
L'archive ouverte pluridisciplinaire HAL, est destinée au dépôt et à la diffusion de documents scientifiques de niveau recherche, publiés ou non, émanant des établissements d'enseignement et de recherche français ou étrangers, des laboratoires publics ou privés. 


\title{
A novel predictive link quality metric for mobile ad-hoc networks in urban contexts
}

\author{
Sebastien Bindel ${ }^{1}$, Serge Chaumette ${ }^{1}$, and Benoit Hilt ${ }^{2}$ \\ 1 LaBRI, University of Bordeaux, Talence, France \\ 2 MIPS, University of Haute Alsace, Colmar, France
}

\begin{abstract}
In Mobile Ad hoc networks (MANETs), advanced routing metrics use Link Quality Estimators (LQE) for making routing decisions. To be efficient and in a strong interaction with the physical layer transmission conditions, the accuracy and the reactivity of LQE used by metrics are crucial for maintaining connectivity. Current LQE estimates the link quality into a single value. This method limits the accuracy of the estimators, especially in highly volatile environments such as VANETs in urban environments. In this paper we propose multi-estimators LQE approach that provides both a better link quality and a link behavior assessment. These novel estimators deal with LQE requirements, reactivity, stability and accuracy to become a reliable LQE. We evaluate they reactivity and accuracy with realistic physical layer and mobility patterns and also found their forecasting properties.
\end{abstract}

Key words: F-ETX, forecasting, routing, link quality

\section{Introduction}

Link quality estimation is one of the main concerns for applications running in Mobile Ad-Hoc Networks (MANET), Vehicular (VANET) and emerging Unmanned System networks. In such networks, the radio signal is affected by several phenomena including shadowing, fading and Doppler effect which degrade its quality. Resulting perturbations contribute to undermine current algorithms, that impact applications performances. For instance, routing protocols often rely on link quality estimations to select reliable links and maintain efficient network operation. Link quality estimation also plays a significant role in topology discovery mechanisms to build a neighborhood knowledge. However the lossy and the dynamic behavior of the links makes this task non trivial.

In the past, several research papers have been focused on the design of link quality estimators and have tackled some received wisdoms. Firstly, empirical studies have shown the absence of correlation between the link quality and the distance between nodes [1]. Secondly, the transitional phase of link quality is often longer than expected and characterized by a significant level of unreliability

\footnotetext{
* The final publication is available at Springer via eudl.eu/doi/10.1007/978-3-31925067-0_11

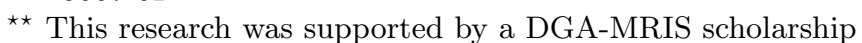


and asymmetry [16]. Thirdly, the correlation between the Packet Reception Ratio (PRR) and the physical information (i.e. RSSI, SNR and LQI) is not easily deductible while these indicators have a limited efficiency to assess the link quality [1] [17] [13]. It is therefore important to take into account these observations to design effective metrics.

Fast ETX (F-ETX) is a novel metric [3] that uses dynamic windows size to sense the link and overcomes intrinsic limitations of static window size and empirical filters such as EWMA. As a result, F-ETX performs better than current ETX based metrics. Even if the metric reacts quickly to persistent changes, transient fluctuations also affects the estimation. F-ETX features make it reactive but instable in some situations. We observed in our experimentations that FETX provides additional information about link features not yet characterized. This paper completes [3] by providing an extended version of F-ETX combining additional companion-metrics to give a multi faced assessment in order to make the metric stable, reactive and accurate. Additionally, it shows the companionmetrics ability to provide reliable predictions of link quality.

The remainder of this paper is divided into five parts. Section 2 reviews current techniques to estimate the quality of a link. Section 3 gives the F-ETX technical background. Section 4 presents the design of the predictive companionmetrics. Section 5 shows a comparative evaluation of our estimators with other metrics. Section 6 concludes this paper and opens up new perspectives.

\section{Related work}

Effective link measurement is a fundamental building block in wireless networks and especially if the propagation channel is subject to important variations. This section summarizes current wireless link quality estimation techniques and point out the main challenges of this hot topic.

\subsection{Logical information as link quality metrics}

Traditional metrics in wired networks, (i.e. hop count) fail to give an estimation on the paths reliability in wireless networks. De Couto et al. have suggested the Expected Transmission Count (ETX) metric [6], which combines forward and backward packet reception to compute a packet delivery success probability. Draves et al [7] have experimented four type of metrics (ETX, hop count, Perhop Round Trip Time and Per-hop Packet Pair Delay) through a test bed placed in an indoor environment. They observed that ETX performs better than the others but in a mobility case, the performance of the hop count metric exceeds the ETX metric. Due to fixed size window based estimation, we showed [3] a short window size leads to a reactive estimation, while a larger window size reduces its agility. The Required Number of Packet (RNP) metric suggested by Cerpa et al [5] performs at MAC layer and counts the average number of packet retransmissions required to deliver it successfully. They observed the temporal 
properties of low power wireless links and described the usefulness of such a metric to measure the link quality compared to reception rate (RR). Unlike the $\mathrm{RR}$ information, RNP metric takes into account the underlying distribution of losses because the link quality is characterized in both directions. However, this metric requires an ARQ (Automatic Repeat-Request) mechanism to retransmit lost packets in order to determine the number of retransmission required to achieve a successful reception. Woo et al [15] designed a routing protocol based on the channel snopping an introduced an agile filter called Window Mean with EWMA (WMEWMA) as an empirical estimator technique [14]. Authors have shown its performance, over the others filters based on the Exponential Weighted Moving Average (EWMA).

\subsection{Physical informations as link quality metrics}

Other metrics, which perform at the PHY layer provide an immediate quality information about the wireless channel. This type of metric has the advantage to be implemented without any additional costs and provides a valuable snapshot of the link state. Such metrics are relied on three kinds of information provided by the PHY layer: the Received Signal Strength Indicator (RSSI), the Signal to Noise Ratio (SNR) and the Link Quality Indicator (LQI). Current works try to establish a correlation between PHY parameters and the PRR but none of them found a satisfactory solution. Zhao et al. [17] showed the difficulty to make a good estimation of the link quality based on RSSI values. Even if a high signal strength can be associated to a high packet reception, the reverse relationship is not true. The use of the SNR was often proposed to overcome RSSI based indicators limitations. However, experimental evaluations have shown that the correlation between the SNR and the PRR is not directly deductible. This is due to the hardware sensitivity and the environmental effects, especially for intermediate link quality [1]. In addition, Boano et al. [4] have shown the limitation of SNR based indicators to differentiate the possible states of a wireless link (transitional and bad), excepted for good links (result confirmed by [13]). The last hardware based metric called LQI has been proposed for the 802.15.4 standard but it can only be exploited on specific radio chips. Even if LQI presents a certain correlation (better than the RSS and closer to the SNR) with the PRR [10], it does not provide a relevant estimation for intermediate link quality [13].

A recent generation of link quality estimators based on the packet decoding process has been proposed. The first one deals with the DSSS decoding error analysis [9] [12] to assess the link quality. The second one proposed by Gabteni et al. [8] uses the OFDM decoding process in order to make an interesting link state indicator which predict future link disruptions. Unfortunately, these estimators are only available on specific radio chips for the first one and only tested in simulations for the second one. 


\subsection{Metrics with multi-estimators}

An interesting novel approach supports that the link assessment can be made with multi-estimators to have a multi-faced vision of the link in order to compute a scored quality link estimation. Baccour et al. [2] designed an hybrid metric based on logical and physical information to provide multi-estimators that assess the packet delivery ratio, the link asymmetry level, the link stability and the channel quality. These estimators are aggregated into a single metric following a fuzzy logic method. The Holistic Packet Statistic (HoPS) metric suggested by Renner et al. [11] incorporates four estimators; namely short-term, long-term, absolute deviation and trend estimation; computed with the EWMA filter. However an intrinsic problem of the use of this filter limits the agility of estimators. It also has the disadvantage to require a large amount of traffic to train the estimators and consequently increases the detection time of link state changes.

\section{F-ETX metric}

The use of a static window size and filters based on EWMA limit the agility of link quality estimators. The F-ETX metric [3] overcomes these limitations by monitoring the link with a dynamic window size. Two windows are maintained, one to estimate the PRR and the second one for the ARR (Acknowledgment Reception Ratio) estimation, respectively represented by $d_{f}$ and $d_{r}$ ratios. Theses windows are also managed by two algorithms; one dealing with their reductions to increase the reactivity of the metric and another one takes care about their growth in order to increase the accuracy and the stability of the metric.

\subsection{Reactivity improvement - Window size reduction}

As reactivity is the major concern for link quality metrics, F-ETX uses an algorithm that ensures both a fresh and an accurate estimation. Each ratio (i.e. $d_{f}$ and $d_{r}$ ) computation uses a binary filter based on the Weighted Moving Average (WMA). It keeps the newest correctly received packet information and penalizes the target ratio according to the current number of lost packets. Let $x_{1} \ldots, x_{1}$ the values representing the current states of reception maintained by a window $W$ at the time $n$. The associated binary weight, $w$, represents the reception state of the $x$ data packet and fixed at 0 for lost and 1 for a good reception. Let $\alpha$ be the number of packets marked as lost in the window and $\left|W_{n}\right|$ the window size at the time $n$. If a loss is detected, the new window size is determined as follows:

$$
\left|W_{n}\right|=\frac{\left|W_{n-1}\right|}{2^{\alpha}}
$$

Th reception probability is computed by:

$$
\text { probability }=\frac{w^{n} x_{n}+w^{n-1} x_{n-1}+\ldots+w^{1} x_{1}}{\left|W_{n}\right|}
$$


As shown in [3], in case of successive packet losses, the algorithm enables an exponential $\left(2^{\alpha}\right)$ decrease of the window size that leads to a rapid link state detection.

\subsection{Accuracy and stability improvement - Growth of the window}

To adapt the link quality assessment according to the link stability, a dedicated algorithm manages the growth of the window size after its reduction. This guarantees a proper estimation of the link quality adjusted to the link stability. When a packet loss occurs, the algorithm saves the current window size, called in the rest of this paper the threshold, $T_{h}$. For each new packet marked as received, the window size is incremented. From the moment the window size reaches the threshold, each new received packet increments a dedicated counter $C_{n}$. Then, the window $(W)$ is increased or shifted (the window is shifted left) under the following conditions.

$$
W\left\{\begin{array}{l}
\text { Increased if } \\
\text { Shifted else }
\end{array} W_{n} \geqslant T_{h} \wedge C_{n} \geqslant \frac{W_{n}}{2}\right.
$$

After each increase, the counter $C_{n}$ is reset. This approach limits the speed of the window size according to the loss occurrence and adjusts the accuracy of the estimation according to the link stability.

\subsection{Discussion}

F-ETX appears as reactive and accurate, but reacts also to transient losses. This makes the metric instable and does not meet requirements to be a suitable LQE. As a matter of fact, reactivity and stability are at odds, a single metric cannot be both reactive and stable. However additional information can be extracted from the metric to assess different link features. This information is fully exploited to provide companion metrics about the link quality trend, the link stability and an indicator about the potential switch from a bidirectional to an unidirectional link. The goals of these companions metrics is to offset the weakness of the link quality assessment and provide additional information on the link states to predict local links features.

\section{Companion estimators of F-ETX}

The F-ETX metric only deals with a single estimation while the algorithm assesses other link features that could be suitable both for local link evaluation and end-to-end path exploration required by routing protocols as example. We argue that a multi-faced representation of the link provides detail information about its state and helps the routing process to select the best suitable link. These estimators are described next. 
Link quality : This estimator is based on the link quality performed by the FETX metric. It uses the forward $d_{f}$ and the backward $d_{r}$ ratios, which represent the probability of a packet to be delivered to a neighbor and the probability of its ACK packet to be successfully received back.

$$
\chi^{L Q}=\frac{1}{\left(1-d_{f}\right)\left(1-d_{r}\right)}
$$

Link quality trend : This indicator tracks the course of the link quality by computing the variation between the current $\chi_{t}^{L Q}$ and the previous estimation $\chi_{t-1}^{L Q}$. To provide a long term estimation the result is averaged with an EWMA.

$$
\begin{aligned}
& \Delta_{t}^{L Q}=\chi_{t}^{L Q}-\chi_{t-1}^{L Q} \\
& \chi_{t}^{T r e n d}=\beta \cdot \Delta_{t}^{L Q}+(1-\beta) \cdot \chi_{t-1}^{T r e n d}
\end{aligned}
$$

The coefficient $\beta$ influences the sensitivity of the filter. Choosing a small $\beta$ value is advisable to achieve a long term estimation. Note that two successives $\chi^{L Q}$ set to 0 indicate a long-term disruption and resets the link quality trend estimator.

Link stability estimation : We observed that a fine analysis of the $d_{f}$ and $d_{r}$ maintained windows content provides a link stability information. Let a binary state $[0,1]$ that represents the reception state of an excepted packet in a window. We note $W_{\max }$ the maximum window size, $W_{n}$ the current window size and the $W_{i}$ the $i^{\text {th }}$ element in the window. The windows maintained to compute the $d_{f}$ and $d_{r}$ probabilities are respectively noted $W^{d_{f}}$ and $W^{d_{r}}$. The link stability indicator is computed with an EWMA filter tacking into account the absolute $\Xi$ and the relative stability $\xi$.

$$
\begin{aligned}
\Xi & =\frac{\sum_{i=1}^{W_{n}^{d_{f}}} W_{i}^{d_{f}}+\sum_{i=1}^{W_{n}^{d_{r}}} W_{i}^{d_{r}}}{2 W_{\max }} \\
\xi & =\frac{\sum_{i=1}^{W_{n}^{d_{f}}} W_{i}^{d_{f}}+\sum_{i=1}^{W_{n}^{d_{r}}} W_{i}^{d_{r}}}{W_{n}^{d_{f}}+W_{n}^{d_{r}}} \\
\chi_{t}^{\text {Stab }} & =\Xi_{t} \cdot \gamma+(1-\gamma) \cdot \xi_{t}
\end{aligned}
$$

The absolute estimation $(\Xi)$ computed from the maximum window size (fixed value) represents the absolute level of stability of the link. The relative estimation $(\xi)$ computed from the current window size (dynamic value) represents the relative stability. This third estimation gives the level of the link stability according to the current window size. This information is useful, since, for a same absolute value, the relative link estimation gives an additional assessment taking into account losses which took place recently. Both absolute and relative information are suitable to characterize the link stability, hence we advise a $\gamma$ value fixed at 0.5 . 
Unidirectional link level : This last estimator deals with the detection of bidirectional links becoming unidirectional. Current approaches like F-LQE with the ASL estimator, track the difference between the uplink and downlink reception rates. Such a method becomes inefficient if the link has a short life time or experiment an high level of packets losses. In this case, windows are not sufficient trained to give a trustworthy estimation. Our method overcomes this limitation by measuring the variation of the up and downlink reception ratios. This makes it independent of the window size and does not require any training period. Let $W$ be a window and $W_{n}^{t}$ its size at time $t$. The variations of the reception ratio provided by the window $W$ at time $t$ is noted $\Delta_{t}^{W i n}$. The indicator is given by:

$$
\begin{aligned}
& \Delta_{t}^{W i n}=\sum_{i=1}^{W_{n}^{t}} W_{i}-\sum_{i=1}^{W_{n}^{t-1}} W_{i} \\
& \chi_{t}^{U L L}=\chi_{t-1}^{U L L} \cdot \lambda+(1-\lambda) \cdot \varphi\left(\Delta_{t}^{d_{f}}, \Delta_{t}^{d_{r}}\right) \\
& \text { with } \varphi(x, y)= \begin{cases}-1 & x<0 \wedge y>0 \\
1 & x>0 \wedge y<0 \\
0 & \text { else }\end{cases}
\end{aligned}
$$

To give a tendency we advise a $\lambda$ value fixed at an high value. When this indicator becomes negative, a link may become unidirectional (e.g. nodes with different transmit power level) .

\section{Evaluation}

This evaluation compares the performances and the robustness of F-ETX and companion metrics to current scored metrics, namely F-LQE and HOPS. Tests were run in an urban environment with both realistic propagation and mobility into the NS-3 simulator. Simulations ran 30 mobile nodes in a $500 \mathrm{~m} \times 500 \mathrm{~m}$ area with realistic urban Manhattan $4 \times 4$ grid based mobility. Nodes have different relative speeds in $[0: 30] \mathrm{m} / \mathrm{s}$. The realistic wireless channel was setup with a ThreeLogDistanceLossModel for modeling shadowing (Exp0: 2.5, Exp1: 5, Exp2: 10, D0: 1m , D1: 75m, D2: 114m ). Fading effect was setup with a RicianPropagationLossModel with a LineOfSightPower fixed at 1. Communications performed with $802.11 \mathrm{~g}$ at $6 \mathrm{Mbp} / \mathrm{s}$. Link quality metrics are measured at the receiver side by monitoring probe packets. Details can be found in [3]. Parameters of LQEs have been set according to [2] for F-LQE and [11] for HoPS.

For F-LQE [2] the parameter of the WMEWMA filter used to determine the packet delivery ratio is set to 0.6 . An history based of $30 \mathrm{PRR}$ is used to compute the link stability and the link quality estimations. Until to reach this threshold, the minimal required history is set to 5 PRR. HoPS [11] uses EWMA filters to compute short- and long-term link quality estimations and their deviation. Coefficients are respectively set to 0.9 and 0.997 and its initializes short- and long-term estimations at $50 \%$ for new links. For F-ETX, parameters $\lambda, \beta$ and $\gamma$ are respectively set to $0.9,0.1$ and 0.5 . To evaluate both the agility of the 


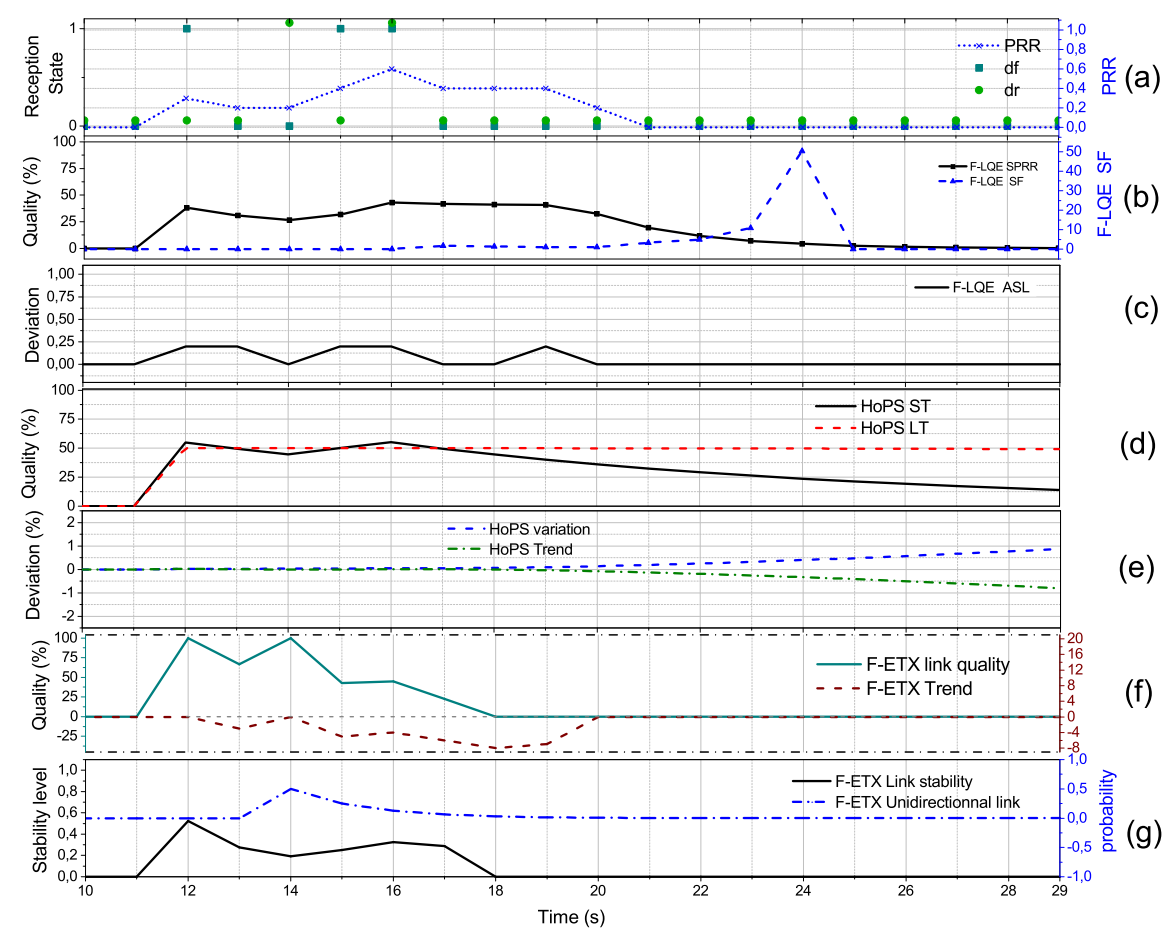

Fig. 1. Fast speed crossing

metrics to track fluctuations of the link quality and the accuracy/robustness of the metrics, we achieved temporal and statistical evaluations.

\subsection{Temporal behavior}

The reliability of metrics were tested under two scenarios; a fast and a slow crossing cases involving two nodes. In the first one, the relative speed is $50 \mathrm{~m} / \mathrm{s}$ and the communication time is $5 \mathrm{~s}$ while in the second one, they are respectively $3 \mathrm{~m} / \mathrm{s}$ and $24 \mathrm{~s}$.

Fast speed scenario According to the distribution of the $d_{r}$ and $d_{f}$ in the figure 1 (a) the link lifetime is very short ( $5 \mathrm{~s}$ between $12 \mathrm{~s}$ and $17 \mathrm{~s}$, while important stochastic losses can be observed resulting from a significant fading (Rayleigh) effect). The PRR computed over an history of 5 packets declares the link disrupted at $21 \mathrm{~s}$, but in fact the disruption occurs at $17 \mathrm{~s}$, in addition a single $d_{f}$ and $d_{r}$ reading is not sufficient to determine the link state. For instance, at $13 \mathrm{~s}$ on a single reading the link could be considered as disrupted, whereas it was a transitory loss (the link remains up until $17 \mathrm{~s}$ ).

About the F-LQE, figure 1(b) shows the smoothed PRR (SPRR) evaluating the link quality and the link stability estimation $(\mathrm{SF})$. Below, the figure 1(c) shows the unidirectionality level of a link estimator (ASL). The SPRR follows 
the corresponding PRR (fig 1) trace with a smoothing trend, but the estimator is clearly not enough reactive and detect the disruption too late. This result from the EWMA filter that gives more important stability than reactivity to the estimator. The SF estimator also suffers from a lag to indicate a decrease of the stability from $22 \mathrm{~s}$, while the link is disrupted, because it is based on an history of five packets to compute the stability estimation. About the ASL indicator, the variation of $d_{r}$ and $d_{f}$ distribution introduces light fluctuations indicating a low probability to have asymmetric link.

Regarding estimators from HoPS (fig 1(d) and (e)) it is observed the slow convergence time of the short-term estimation, while a link is disrupted, the estimator declares the link quality as not disrupted. But the long-term estimator indicates a correct trend of decrease. Consequently, the EWMA filter is well used for long-term estimation but not suitable for short-term estimation which too smooth estimations. In the same manner, the link quality trend and the variation indicators are affected by the long reactivity of the short estimation and react too slowly when a disruption occurs .

The estimators of F-ETX are shown figure 1(f) and (g). In contrast with LQE, F-ETX is more reactive than the others and declares the link disrupted earlier (at 18s). The trend estimation indicates a degradation of the link quality via consecutive negative values. This is confirmed by the link stability estimator indicating a low level of stability and a decrease. About the unidirectional indicator, its gives a false positive value (at 14s) indicated that the link can be unidirectional in the opposite direction.

Slow speed scenario The distribution of $d_{r}$ and $d_{f}$ and the PRR indicate a progressive increase of the link quality, fig $2(\mathrm{a})$.

Concerning F-LQE, the SPRR (fig 2(b)) progressively increases and confirms this tendency. The stability is correctly estimated by the SF estimator, indicating a decrease of the stability at the beginning of the communication and a progressively increase for the rest of the simulation. About the ASL, stochastic losses disturb the estimator (fig 2(c)), that indicates the possibility of the link to be unidirectional.

About HoPs the short-term link quality estimators also indicates an improvement of the link quality and is confirmed by the long-term indicator that progressively increases (fig 2(d)). We note that the HoPS ST increases faster than HoPS LT. But the HoPS trend and variation (fig 2(e)) take the same course indicating an increase of the link stability and a variation between HoPs ST and LT.

Regarding F-ETX (fig 2(f) and (g)) the link quality estimation also increases, this is confirmed by the trend estimators indicating a decrease at the beginning, but progressively indicates an improvement of the link quality. The same observation can be pointed out on the link stability indicator. Unlike the ASL indicator the link unidirectionality level estimator of F-ETX varies a little and reflects the constant bidirectional property of the link. 


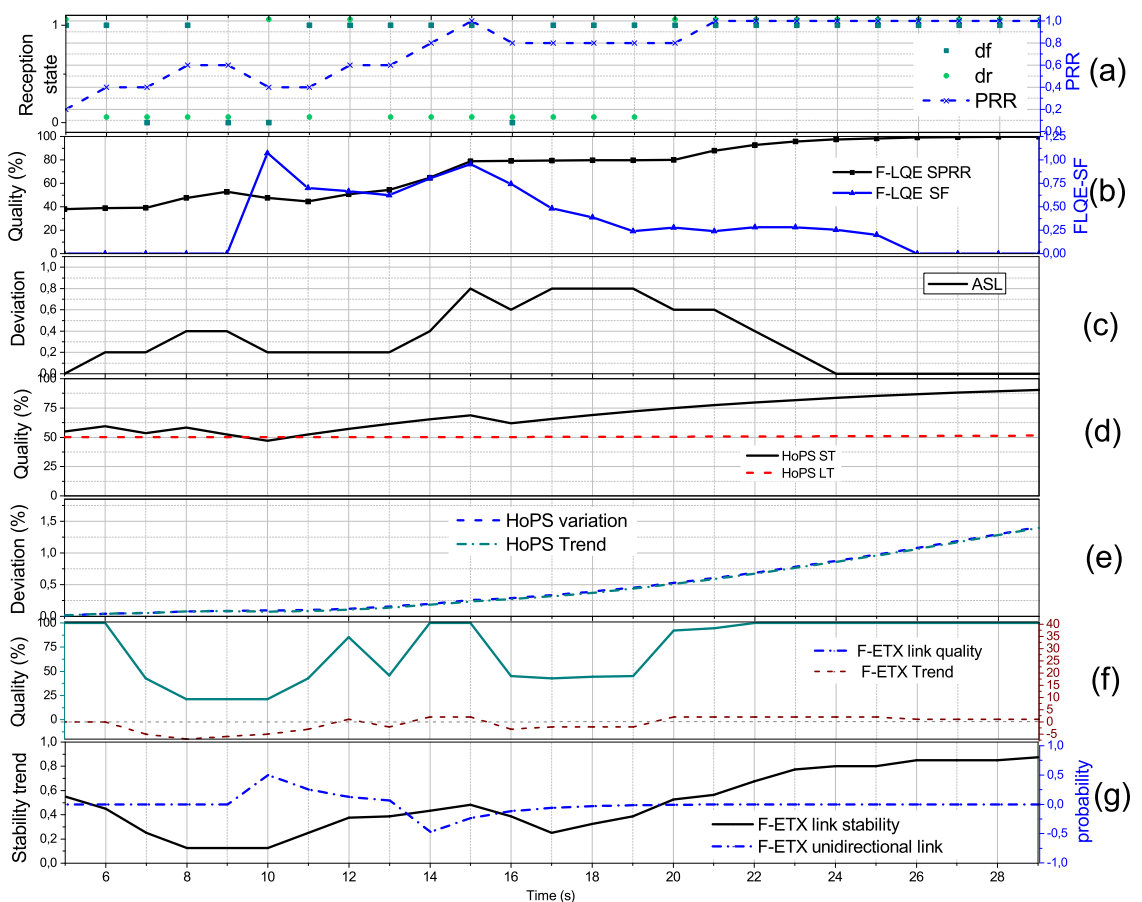

Fig. 2. Slow speed crossing

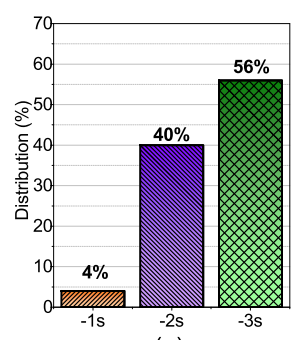

(a)

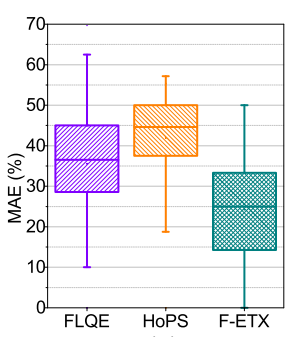

(b)

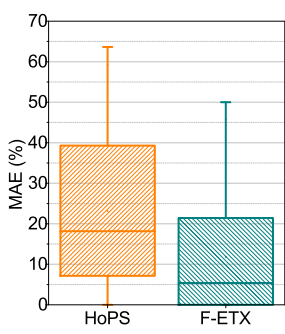

(c)

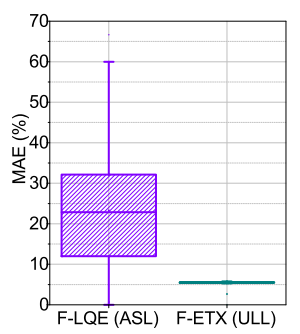

(d)

Fig. 3. Statistical analysis

\subsection{Statistical evaluation}

While previous evaluations give a detail about the strength and weakness of LQEs, this evaluation is extended with statistical analysis from all links of the simulation. Over 470 links have been analysed where 1800 packets have been exchanged.

Previously we have observed that the link quality estimator of F-ETX is more reactive than F-LQE and HoPs. In this statistical study we are focus on how this estimator can anticipate disruption compared to the PRR solution based on 
an history of 5 packets. Figure 3 (a) shows that F-ETX is clearly the best solution by anticipated disruption before the PRR solution. Because based on the dynamic window size the metric is more reactive and track very well link states changes. In addition, F-ETX assess both link direction unlike a PRR solution that only evaluates downlink.

The rest of the statistical analysis is made with the Mean Absolute Error (MAE) that measures the magnitude of the predicted estimation and the current outcome. A low score indicates a good prediction while a bigger value indicates a greater error between the prediction and the current value. Tracking link stability is an essential feature to detect and differentiate transient and persistent link. We have compared Fig 3 (b) the variation of the value given by these estimators to the current variation observed from the delivery and forward ratios. F-ETX estimator gives the lowest MAE compared to the other. Because HoPS indicator only tracks the variation between the HoPs ST and LT estimations, that not really related with the link stability and about F-LQE, the estimation is based on a PRR history that produce a lag generating consecutive error predictions. The link quality trend is a complementary information that determines the current course of the link quality. Fig 3 (c) shows the link quality trend of HoPs and F-ETX. We observe the better ability of our estimator to give the tendency of the link quality compared to HoPS. Even if each of them is based on the link quality estimator and both are computed with an EWMA filter. Their abilities to track the link quality course depends on the ability of link quality estimator, as HoPS-ST suffers from lag with the use EWMA filter impacting the HoPS-LT. On the other hand, the link quality estimator from F-ETX is react but instable. That is why with the use of EWMA the estimation is stabilized given a better long-term estimation than the HoPS-LT overestimating the tendency.

Figure 3 (d) shows the unidirectional link estimator of the F-LQE and F-ETX. During the simulation, any effective unidirectional link are present. ASL estimation only made a single reading on the reception ratio of the up and downlink often different when highly propagation disturbances are present. Our indicator adopts another strategy based on the variation between the up and downlink, this results that our estimation is more robust to disturbance and give more suitable information about the potential of a bidirectional link to become unidirectional.

\section{Conclusion}

In this paper we presented novel companion-metrics of our recently proposed F-EXT metric [3]. Each of them evaluates a specific link feature; (i) the link quality, (ii) its trend, (iii) its stability and (iv) the detection of unidirectional links. They do not require a training period and are computed efficiently by the use of dynamic sized windows and moving average filters. These four metrics have then be compared to popular propositions of LQEs from the literature (e.g. F-LQE and Hops). Comparisons took two forms. First showed the capacity of the metrics to track accurately link disruption and link upcoming in two nodes 
crossing scenarios (e.g. a fast $50 \mathrm{~m} / \mathrm{s}$ and an slow $3 \mathrm{~m} / \mathrm{s}$ one). They also highlighted prediction capabilities of the metrics. The second evaluation confirmed this prediction capability through a statistical analysis that show how F-ETX and its companion-metrics overcomes other LQE when dealing with link disruption forecasting. The capability to detect a wireless link that switches from bidirectional to unidirectional was also tested. To test the robustness of the new metrics, simulations ran realistic channel propagation models and realistic urban mobility. This work shows two important elements relating to link quality monitoring; to track efficiently a link expecting volatile channel propagation conditions, the use of dynamic sized windows overcomes the classical methods, a metric associated to companion-metrics allows a better assessment of channel properties and is therefore more suitable for usage into higher levels.

This is precisely the aim of our future works. We plan to test the F-ETX and companion-metrics in other contexts such as building environments to confirm the suitability of our approach. We will also integrate the F-ETX and companionmetrics into routing protocols to test their efficiency in multi-hop path setup.

\section{References}

1. Baccour, N., Koubâa, A., Mottola, L., Zúñiga, M., all: Radio link quality estimation in wireless sensor networks: A survey. ACM TOSN (2012)

2. Baccour, N., Koubâa, A., Youssef, H., Ben Jamãa, M., do Rosario, D., Alves, M., Becker, L.: F-LQE: A fuzzy link quality estimator for wireless sensor networks. In: Wireless Sensor Networks. Springer Berlin Heidelberg (2010)

3. Bindel, S., Chaumette, S., Hilt, B.: F-ETX: an enhancement of ETX metric for wireless mobile networks. In: Nets4Cars (2015)

4. Boano, C., Zúñiga, M., Voigt, T., Willig, A., Romer, K.: The triangle metric: Fast link quality estimation for mobile wireless sensor networks. In: IEEE ICCCN (2010)

5. Cerpa, A., Wrong, J., Potkonjak, M., Estrin, D.: Temporal properties of low power wireless links: modeling and implications on multi-hop routing. In: ACM MobiHoc (2005)

6. De Couto, D., Aguayo, D., Bicket, J., Morris, R.: A high-throughput path metric for multi-hop wireless routing. In: ACM MobiCom (2003)

7. Draves, R., Padhye, J., Zill, B.: Comparison of routing metrics for static multi-hop wireless networks. In: ACM SIGCOMM (2004)

8. Gabteni, H., Hilt, B., Drouhin, F., Ledy, J., Basset, M., Lorenz, P.: A novel predictive link state indicator for ad-hoc networks. In: IEEE Globecom (2014)

9. Heinzer, P., Lenders, V., Legendre, F.: Fast and accurate packet delivery estimation based on DSSS chip errors. In: IEEE INFOCOM (2012)

10. Liu, T., Cerpa, A.: Data-driven link quality prediction using link features. In: ACM TOSN (2014)

11. Renner, C., Ernst, S., Weyer, C., Turau, V.: Prediction accuracy of link-quality estimators. In: ACM EWSN (2011)

12. Spuhler, M.and Lenders, V., Giustiniano, D.: BLITZ: Wireless link quality estimation in the dark. In: Wireless Sensor Networks. Springer Berlin Heidelberg (2013)

13. Srinivasan, K., Kannan, D., Tavakoli, A., Philip, L.: An empirical study of lowpower wireless. ACM TOSN (2010) 
14. Woo, A., Culler, D.: Evaluation of efficient link reliability estimators for low-power wireless networks. Tech. Rep. UCB/CSD-03-1270, EECS Department, University of California, Berkeley (2003)

15. Woo, A., Tong, T., Culler, D.: Taming the underlying challenges of reliable multihop routing in sensor networks. In: ACM Senys (2003)

16. Zamalloa, M., Krishnamachari, B.: An analysis of unreliability and asymmetry in low-power wireless links. ACM TOSN (2007)

17. Zhao, J., Govindann, R.: Understanding packet delivery performance in dense wireless sensor networks. In: ACM Sensys (2003) 